\title{
EVALUACIÓN PRELIMINAR DE ORQUÍDEAS EN EL PARQUE NACIONAL CUTERVO, CAJAMARCA-PERÚ
}

\author{
PRELIMINARY ASSESSMENT OF ORCHIDS IN THE NATIONAL PARK \\ CUTERVO, CAJAMARCA - PERÚ
}

\author{
María Sánchez Recuay ${ }^{1}$ y Abelardo Calderón Rodríguez ${ }^{2}$
}

\begin{abstract}
Resumen
En el departamento de Cajamarca, los llamados "bosques húmedos de ceja de selva" (caracterizados por su gran complejidad florística, centros de endemismo y gran variedad de orquídeas) albergan a los "bosques de la Cordillera de Tarros del Parque Nacional Cutervo". Este primer Parque Nacional del Perú creado en 1961, sufre actualmente la explotación irracional y destrucción de sus bosques, consecuencia de la actividad extractiva de madera y la ampliación de la frontera agrícola y ganadera. Las potencialidades y riesgos presentes en estos bosques, además del escaso conocimiento respecto a la familia Orchidaceae, motivaron el presente estudio preliminar de la riqueza y distribución de orquídeas, a través del muestreo por cuadrantes y transectos, durante los meses de febrero y marzo del año 2004. Pese a la difícil identificación de las especies encontradas, por no ser la época característica de floración de orquídeas, se pudo registrar un total de 88 morfoespecies, identificándose el 63.6 \%, repartidos en 16 géneros, de los cuales Pleurothalis, Epidendrum, Maxillaria y Stelis mostraron ser los géneros más diversos y frecuentes dentro del área de estudio.
\end{abstract}

Palabras clave: orquídeas, Parque Nacional Cutervo, Cordillera los Tarros

\begin{abstract}
In the department of Cajamarca, the so-called "cloud forests of the tropical rainforest" (characterized by their great variety of orchids, floristic complexity and endemism centers) are home to the "Cordillera de los Tarros" Forests, inside Cutervo National Park. This first National Park created in 1961 in Peru, is currently experiencing the irrational exploitation and destruction of its forests, as a consequence of logging activities and the expansion of agricultural and livestock frontiers. The potentials and risks in these forests, as well as scarce knowledge on the Orchidaceae family, prompted this preliminary study on richness and distribution of orchids using square sampling plots and transects, during the months of February and March, 2004. Despite the difficult identification of the species that were found, due to the fact that it was not the characteristic flowering time for the orchids, a total of 88 morphospecies were found, $63.6 \%$ were identified and distributed into 16 genera, of which Pleurothalis, Epidendrum, Maxillaria and Stelis proved to be the most diverse and frequent in the study area.
\end{abstract}

Key words: Orchids, Cutervo National Park, Los Tarros Mountain Range

\section{Introducción}

Las orquídeas constituyen una numerosa familia de plantas con flores. Según los estudios de paleobotánica, se estima que la familia Orchidaceae existe desde hace 120 millones de años. Es una de las familias más evolucionadas y numerosas entre las plantas superiores, debido a su alto grado de diversidad y complejidad en la formación de cada una de sus especies. Cuenta con alrededor de 25000 especies descritas, repartidas en 750 géneros (Cavero et al., 1991), que se encuentran distribuidas por todo el mundo adaptadas a diversos hábitats, en altitudes que van desde los 100 a 4 800msnm., y tan solo ausentes en la Antártida y en los desiertos mas áridos de la tierra. Son plantas herbáceas que muestran singulares características morfotaxonómicas de evolución avanzada. Se las encuentra creciendo sobre piedras (litofíticas), sobre árboles (epifitas), debajo de la tierra (las subterráneas de Australia que solo ven el sol cuando florecen como Rhizanthella gardneri), y en tierra (las plantas terrestres del género Sobralia); además es posible hallar orquídeas con flores desde los dos milímetros, como Trizeuxis falcata, hasta los 70 centímetros (como Phragmipedium caudatum, la flor más grande); sin embargo, tanto en unas como en otras, se puede reconocer un modelo estructural único.

De acuerdo a los estudios de Bennett \& Christenson (1993, 1995), el Perú tiene alrededor de tres mil especies de orquídeas, distribuidas desde Tumbes hasta Puno, en lomas costeras, en regiones 
montañosas occidentales y orientales de los Andes hasta la llanura Amazónica.

La mayor variedad de orquídeas se encuentra en las formaciones de "bosques húmedos de ceja de selva”, comprendidos entre los 500 y 3 600msnm, caracterizados por su gran complejidad florística en sus diferentes formas de vida (epifitas, hierbas, lianas, arbustos y árboles) y donde se han registrado muchos centros de endemismo. En el departamento de Cajamarca se han descrito algunos de estos ecosistemas, por ejemplo los bosques de Podocarpus, que son considerados como la zona más rica en orquídeas, muchas de las cuales son consideradas exóticas como Masdevallia setacea, Masdevallia glandulosa y Lycaste denningiana y los "bosques de la Cordillera de Tarros del Parque Nacional Cutervo”, Área Natural Protegida (ANP), donde por el contrario no se han realizado estudios específicos sobre esta familia, tan solo se han descrito algunas especies de orquídeas, por antiguas exploraciones, cuando las vías de comunicación eran muy escasas, por lo que se sabe poco respecto a la diversidad real de orquídeas en esta zona de conservación.

El presente trabajo es un estudio preliminar de orquídeas del Parque Nacional Cutervo, con la finalidad de registrar la riqueza de especies y su distribución en la zona de estudio, además de estimar su abundancia y registrar sus diferentes tipos de hábitats. La destrucción de los bosques por la extracción de madera y ganadería son razones suficientes que motivan a realizar la presente investigación.

\section{Materiales y métodos}

El estudio se llevó acabo en el año 2004, en el área comprendida por el Parque Nacional Cutervo (PNC), que fue establecido el 8 de septiembre de 1961 con una extensión de 2500 ha, y en el área comprendida por su propuesta de ampliación, de 8214.23 has de extensión, la cual fue posteriormente aprobada en el año 2006 (Ley No. 28860 SINANPE). La extensión inicial del PNC estuvo comprendida entre los $6^{\circ} 8^{\prime}$ y $6^{\circ} 19^{\prime}$ Latitud Sur y los $78^{\circ} 42^{\prime}$ y $78^{\circ} 51^{\prime}$ Longitud Oeste, y la propuesta de ampliación estuvo dividida en dos sectores: Sector Norte con 2429.54 ha (distritos de Santa Cruz, Pimpingos, Santo Tomás y San Andrés de Cutervo) y el Sector Sur con 5784.69 ha. (distritos de Callayuc, San Andrés de Cutervo, Santo Domingo de la Capilla y Santo Tomás); entre los 1500 y 3500 msnm, comprendiendo los bosques de la Cordillera de Tarros (hoya del Marañón) de la provincia de Cutervo en el departamento de Cajamarca.

Esta ANP cuyo objetivo es el de conservar la flora, fauna y bellezas escénicas de la Cordillera de Tarros, está caracterizada por el bosque húmedo tropical que según el sistema de clasificación Holdridge, comprende las formaciones denominadas Bosque húmedo montano bajo tropical (Bh-MBT) y Bosque muy húmedo montano tropical (Bmh-MT), además de formaciones ecológicas como la puna hasta 3500 msnm, el bosque seco ecuatorial en el norte hasta los 2 200msnm, los bosques orientales de tierras más bajas que se encuentran cerca de los $1200 \mathrm{~m}$ y una serie de ambientes de humedales asociados a los ríos y pequeños lagos del área, que muestra un paisaje de cadenas montañosas bastante accidentada, con pendientes que varían de 10 a 100\%.

En la zona predomina un clima húmedo a semifrío, originado por las neblinas provenientes de la Amazonía que invaden el bosque diariamente durante todo el año, lo que aumenta las precipitaciones de 800 mm (precipitación anual promedio) a más de 2000 $\mathrm{mm}$. Las temperaturas varían entre los $12.5^{\circ} \mathrm{C}$ y $19^{\circ} \mathrm{C}$, con descensos entre noviembre y abril, época de precipitaciones pluviales constantes y neblina persistente, pero sin notables diferencias de temperatura entre el día y la noche en los meses de mayo a octubre.

La flora de estos bosques es extremadamente rica y variada en especies medicinales, maderables y ornamentales, clasificadas en 125 familias, 340 géneros y 683 especies estudiadas. Entre ellas destacan, Ceroxilon andicola "palmera blanca", considerada la especie símbolo del Parque Nacional Cutervo, Cyathea asperata “chontilla”, Winmania sp. "zallyu”, Juglans neotropica "nogal”, Cinchona officinalis “cascarilla”, Cedrela ssp. "cedro blanco” y "cedro colorado", Piptadenia sp. "huayo", Alnus acuminata "aliso", Nectandra sp. "roble”, Podocarpus oleifolius "saucecillo"; el liquen Physma peruvianum, especie endémica del área, y 23 géneros de orquídeas, según López (1971), dentro de los que destacan Epidendrum, Pleurothallis, Odontoglosum y Oncidium, con un mayor número de especies.

\section{Unidades de muestreo}

En el presente trabajo se emplearon como unidades de muestreo para la evaluación de orquídeas las Parcelas de Monitoreo de Flora Silvestre y los transectos de Evaluación de Avifauna Silvestre, establecidas en 16 subsectores comprendidos dentro del PNC y de la zona propuesta para su ampliación, para el monitoreo mensual de la Flora y Fauna Silvestre, llevada a cabo desde el año 2000 por el personal de guardaparques del ANP. Estas unidades de muestreo son permanentes y, están clasificadas de acuerdo a la sectorización del área (Tabla 1).

Se evaluaron siete parcelas o cuadrados de 50x50 $\mathrm{m}^{2}$, que fueron establecidas al azar en los subsectores de Las Grutas, Shitabamba y Pindoé comprendidas dentro del PNC, y los subsectores de San Pedro, Cedropampa, Calabocillo y Bañadera del Oso de la zona propuesta para la ampliación del PNC. Además, se evaluaron 22 transectos lineales de aproximadamente $200 \mathrm{~m}$ de longitud, los cuales fueron trazados por pares y de manera preferencial, de tal manera que uno fue establecido dentro de una 
Tabla 1. Sectorización de las Unidades de Muestreo dentro del Área de estudio.

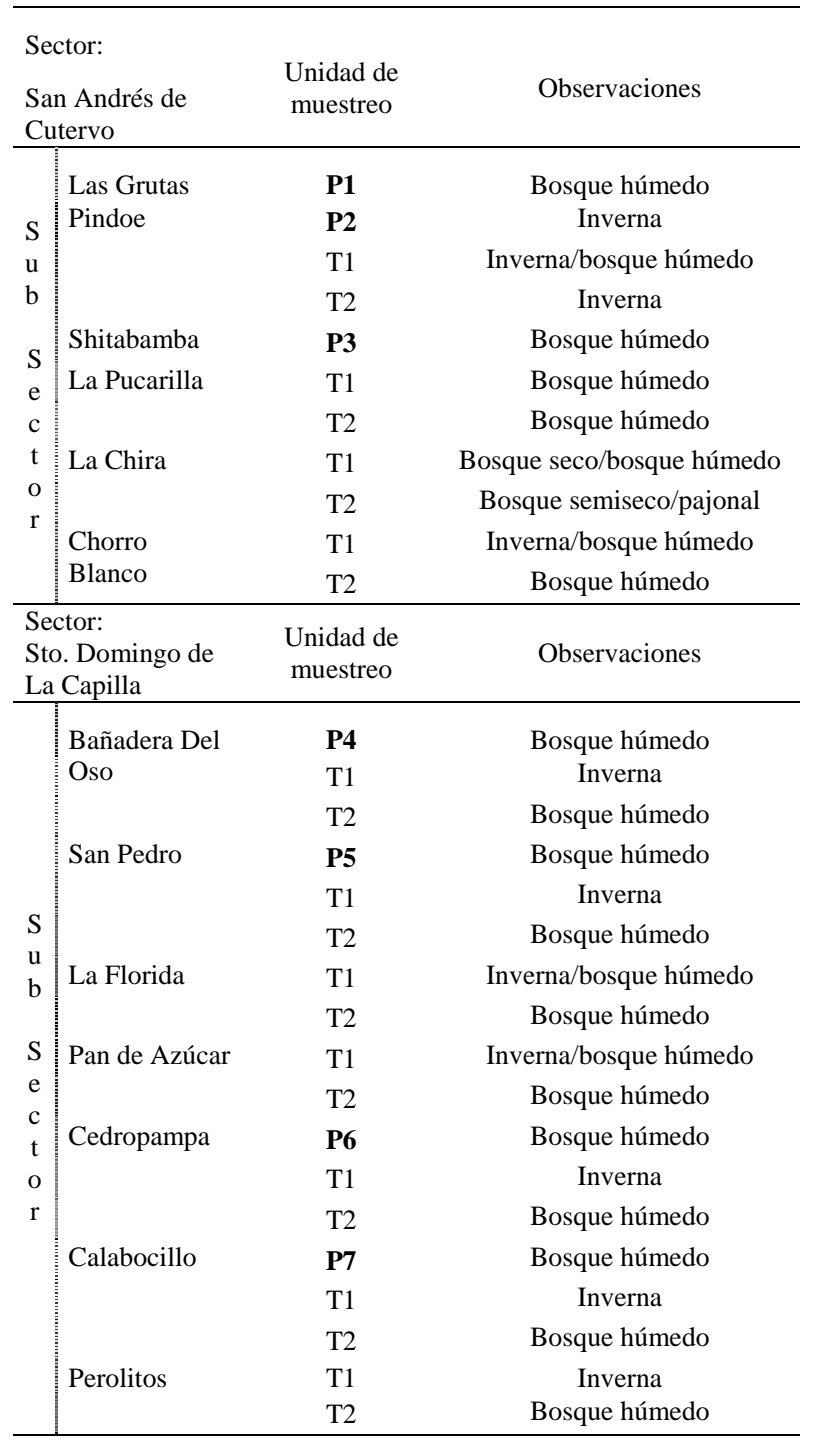

P : Parcela; $\mathbf{T}$ : Transecto.

Inverna: Sector de pastizal, para el pastoreo de ganado vacuno, con algunos árboles maduros.

inverna y su par respectivo fue establecido en el área de bosque adyacente. Los 11 pares de transectos se distribuyen en los subsectores de La Chira, La Pucarilla, Pindoé y Chorro Blanco comprendidos dentro del área del PNC, y los subsectores de Bañadera del Oso, San Pedro, Cedropampa, La Florida, Pan de Azúcar, Calabocillo y Perolitos de la zona propuesta para la ampliación del PNC.

Evaluación en campo, registro de datos e identificación de orquídeas.

La evaluación de orquídeas en campo se llevó a cabo durante los meses de febrero y marzo de 2004, aprovechando las actividades y salidas de monitoreo de flora y avifauna ejecutada por los guardaparques del ANP. Durante estas salidas al campo se emplearon los materiales básicos necesarios como son una vestimenta adecuada para las condiciones de lluvia (botas e impermeable, indispensables), binoculares (Olympus 12x50), cámara fotográfica (Pentax ESPIO 120SW), altímetro (Brunton Sherpa-Switzerland), libreta de campo, etiquetas, lápiz, bolsas, etc.

Cada unidad de muestreo fue recorrida a pie, siguiendo un patrón en zigzag dentro de los cuadrados, y un patrón de recorrido lineal con una cobertura visual de $5 \mathrm{~m}$ a ambos lados para los transectos, evaluándose las orquídeas ubicadas a nivel del sotobosque de fácil observación y accesibilidad, hasta unos $6 \mathrm{~m}$ de altura aproximadamente, encontradas sobre árboles vivos, ramas, troncos o árboles caídos.

Las orquídeas encontradas se clasificaron según su diferenciación vegetativa o florística considerándolas como morfoespecies. Cada morfoespecie fue codificada con las siglas del nombre de los colectores y un número asignado según su colecta desde el inicio de la evaluación. Se registraron los datos de la unidad de muestreo, la fecha, la altitud, la fenología, el crecimiento, el árbol hospedero, el hábitat y la abundancia cualitativa; además, de fotografías.

Las orquídeas encontradas en áreas taladas de bosque (algunas dentro de las unidades de muestreo), que no podrían sobrevivir en tales condiciones de destrucción de su hábitat, fueron colectadas como muestra botánica viva para constituir un orquideario en la sede San Andrés del PNC. Las plantas que se encontraron en estado de floración fueron revisadas para su determinación, empleando manuales ilustrativos de determinación de especies de orquídeas, las flores de las plantas no identificadas, fueron conservadas en alcohol y revisadas posteriormente, durante los meses de abril y mayo, en la Universidad Nacional de San Martín y en la Universidad Nacional Agraria La Molina, determinándoselas hasta el nivel de género.

Estimación de la abundancia de especies.

Se evaluaron dos de los parámetros referidos a la abundancia: la densidad y la frecuencia.

Densidad de especies.

La densidad de una especie en una comunidad convencionalmente corresponde al número de individuos por unidad de superficie, no obstante puede expresarse también de acuerdo a una escala cualitativa como la escala nominal, por lo cual se consideró una escala de cinco categorías nominales: 1-rara, 2ocasional, 3-frecuente, 4-abundante y 5-muy abundante, señalada por Tansley \& Chipp (Graf \& Sayagués, 2000) para el registro de la densidad de especies en una comunidad. La densidad fue estimada para cada morfoespecie de orquídea, a través de la observación en campo de la recurrencia de la morfoespecie dentro del área de cada unidad de muestreo donde fue encontrada. 
Tabla 2. Géneros y especies de orquídeas.

\begin{tabular}{|c|c|c|c|c|c|c|c|c|}
\hline SUB SECTOR & ESPECIE & UBICACIÓN & DENSIDAD & $\begin{array}{l}\text { ALTITUD } \\
\text { (m.s.n.m.) }\end{array}$ & $\mathrm{T}(\mathrm{oC})$ & HABITO & FENOLOGIA & $\begin{array}{c}\text { SUSTRATO } \\
\text { /HOSPEDERO }\end{array}$ \\
\hline PINDOE & Barbosella cucullata & P2 & 1 & 2861 & 19 & Ер & $\mathrm{Fl}$ & tronco caído \\
\hline LA CHIRA & Barbosella sp. 1 & $\mathrm{~T} 2$ & 1 & 2538 & 12 & Еp & $\mathrm{Fl}$ & tronco caído \\
\hline LA CHIRA & Elleanthus estrobillifer & $\mathrm{T} 1$ & 5 & 2554 & 13 & $\mathrm{Te}$ & $\mathrm{Fl}$ & suelo rocoso \\
\hline LA CHIRA & Elleanthus sp. 1 & $\begin{array}{l}\text { Laguna } \\
\text { La Chira }\end{array}$ & 5 & 2703 & 14 & $\mathrm{Te}$ & $\mathrm{Fl}$ & suelo arcilloso \\
\hline PEROLITOS & Epidendrum secundum & $\begin{array}{l}\text { Ruinas } \\
\text { Perolitos }\end{array}$ & 2 & -- & -- & $\mathrm{Te}$ & $\mathrm{Fl}$ & suelo arcilloso \\
\hline LAS GRUTAS & Epidendrum sp. 1 & P1 & 1 & 2848 & 12 & Ер & $\mathrm{Fl}$ & "huayo" Piptadenia sp. \\
\hline LAS GRUTAS & Epidendrum sp. 2 & P1 & 1 & 2848 & 12 & Ep & $\mathrm{iFl}$ & "huayo" Piptadenia sp. \\
\hline PINDOE & Epidendrum sp. 3 & $\mathrm{~T} 1$ & 1 & 2832 & 19 & Еp & $\mathrm{Fl}$ & "huayo" Piptadenia sp. \\
\hline LA CHIRA & Epidendrum sp. 4 & $\mathrm{~T} 2$ & 3 & 2490 & 12 & $\mathrm{Te}$ & $\mathrm{Fl}$ & suelo arcilloso \\
\hline SHITABAMBA & Epidendrum sp. 5 & P3 & 4 & 2828 & 15 & Еp & $\mathrm{iFl}$ & "juangil" caído \\
\hline CHORRO BLANCO & Epidendrum sp. 6 & $\begin{array}{l}\text { Catarata } \\
\text { Chorro } \\
\text { Blanco }\end{array}$ & 1 & 2197 & 19 & Ep & $\mathrm{Fl}$ & "silvana" \\
\hline LA FLORIDA & Epidendrum sp. 7 & $\mathrm{~T} 1$ & 3 & 2311 & 14 & Ер & $\mathrm{Fl}$ & tronco caído \\
\hline LA FLORIDA & Epidendrum sp. 8 & $\mathrm{~T} 1$ & 3 & 2256 & -- & Ep & $\mathrm{fFl}$ & "roble" Nectandra sp. \\
\hline CALABOCILLO & Epidendrum sp. 9 & P7 & 3 & 2189 & 15 & Еp & $\mathrm{Fl}$ & "zallyu" Winmania sp. \\
\hline LAS GRUTAS & Fernandezia subbiflora & P1 & 2 & 2500 & & Ep & $\mathrm{Fl}$ & tronco caído \\
\hline SHITABAMBA & Fernandezia ionanthera & P3 & 4 & 2835 & 15 & Ep & $\mathrm{Fl}$ & "zallyu" Winmania sp. \\
\hline PAN DE AZUCAR & Lockharthia sp. 1 & $\mathrm{~T} 2$ & 2 & 2200 & 14 & Ep & iFl & "roble" Nectandra sp. \\
\hline CHAQUIMAYO & Lycaste sp. 1 & Quebrada & 2 & -- & -- & Еp & $\mathrm{fFl}$ & "cedro" Cedrela ssp. \\
\hline CALABOCILLO & Masdevallia sp. 1 & P7 & 3 & 2158 & 15 & Ep & iFl & "zallyu" Winmania sp. \\
\hline CEDROPAMAPA & Maxillaria huancabambae & $\mathrm{T} 2$ & 2 & 2497 & 14 & Ep & $\mathrm{Fl}$ & "lanchesillo" \\
\hline LAS GRUTAS & Maxillaria sp. 1 & P1 & 3 & 2888 & 14 & Еp & $\mathrm{Fl}$ & "huayo" Piptadenia sp. \\
\hline LAS GRUTAS & Maxillaria sp. 2 & P1 & 1 & -- & -- & Ep & $\mathrm{Fl}$ & tronco caído \\
\hline PINDOE & Maxillaria sp. 3 & $\mathrm{~T} 1$ & 3 & 2823 & 19 & Еp & $\mathrm{iFl}$ & "huayo" Piptadenia sp. \\
\hline PINDOE & Maxillaria sp. 4 & T1 & 2 & -- & -- & Ep & $\mathrm{Fl}$ & "huayo" Piptadenia sp. \\
\hline LA CHIRA & Maxillaria sp. 5 & T1 & 1 & 2554 & 12 & Ep & $\mathrm{Fl}$ & "lalush" \\
\hline SHITABAMBA & Maxillaria sp. 6 & P3 & 1 & 1818 & 15 & Ep & $\mathrm{Fl}$ & "zallyu" Winmania sp. \\
\hline LA FLORIDA & Maxillaria sp. 7 & $\mathrm{~T} 2$ & 2 & 2270 & & Ep & $\mathrm{fFl}$ & tronco caído \\
\hline PAN DE AZUCAR & Maxillaria sp. 8 & $\mathrm{~T} 2$ & 3 & 2207 & 14 & Ep & $\mathrm{Fl}$ & "matico" Piper sp. \\
\hline PAN DE AZUCAR & Maxillaria sp. 9 & $\mathrm{~T} 2$ & 3 & 2207 & 14 & Еp & $\mathrm{fFl}$ & "matico" Piper sp. \\
\hline CALABOCILLO & Maxillaria sp. 10 & P7 & 2 & 2191 & 14 & Ep & $\mathrm{Fl}$ & tronco caído \\
\hline CALABOCILLO & Maxillaria sp. 11 & P7 & 3 & 2191 & 14 & Ep & $\mathrm{Fl}$ & tronco caído \\
\hline CHORRO BLANCO & Odontoglosum sp. 1 & $\mathrm{~T} 1$ & 3 & 2460 & 18 & Ep & $\mathrm{Fl}$ & tronco caído \\
\hline EL SURO & Odontoglosum sp. 2 & Inverna & 1 & 2650 & 17 & Еp & $\mathrm{Fl}$ & tronco caído \\
\hline LA PUCARILLA & Oncidium macranthum & $\mathrm{T} 2$ & 3 & 2900 & 14 & Ep & $\mathrm{Fl}$ & tronco caído \\
\hline SHITABAMBA & Otoglosum sp. 1 & P3 & 3 & 2847 & 15 & Еp & $\mathrm{iFl}$ & "zalluff", "juangil" \\
\hline SHITABAMBA & Pleurothallis flexuosa & P3 & 3 & 2847 & 15 & Еp & $\mathrm{Fl}$ & "juangil" \\
\hline LAS GRUTAS & Pleurothalis sp. 1 & P1 & 3 & -- & -- & Ер & $\mathrm{Fr}$ & tronco caído \\
\hline PINDOE & Pleurothalis sp. 2 & P2 & 3 & 2849 & 19 & Ep & $\mathrm{fFl}$ & tronco caído \\
\hline LA CHIRA & Pleurothalis sp. 3 & $\mathrm{~T} 1$ & 1 & 2528 & 12 & Ep & iFl & tronco caído \\
\hline SHITABAMBA & Pleurothalis sp. 4 & P3 & 3 & 1818 & 15 & Ep & $\mathrm{Fl}$ & "zallyu" Winmania sp. \\
\hline SHITABAMBA & Pleurothalis sp. 5 & P3 & 3 & 2835 & 15 & Еp & iFl & hojarasca \\
\hline CHORRO BLANCO & Pleurothalis sp. 6 & $\begin{array}{l}\text { Catarata } \\
\text { Chorro } \\
\text { Blanco }\end{array}$ & 1 & 2190 & 16 & Ep & iFl & tronco caído \\
\hline CEDROPAMAPA & Pleurothalis sp. 7 & T1 & 2 & 2346 & 14 & Ер & $\mathrm{iFl}$ & tronco caído \\
\hline CALABOCILLO & Pleurothalis sp. 8 & P7 & 4 & 2161 & 14 & Ep & $\mathrm{fFl}$ & "zallyu" Winmania sp. \\
\hline CALABOCILLO & Pleurothalis sp. 9 & P7 & 3 & 2189 & 15 & Ep & $\mathrm{Fl}$ & "zallyu" Winmania sp. \\
\hline CALABOCILLO & Pleurothalis sp. 10 & P7 & 3 & 2189 & 15 & Ep & $\mathrm{Fl}$ & "zallyu" Winmania sp. \\
\hline PEROLITOS & Pleurothalis sp. 11 & $\mathrm{~T} 2$ & 2 & -- & -- & Еp & $\mathrm{iFl}$ & tronco caído \\
\hline $\begin{array}{l}\text { LA FLORIDA, } \\
\text { CEDROPAMAPA }\end{array}$ & Sobralia rosea & Quebrada & 4 & 1914 & -- & $\mathrm{Te}$ & $\mathrm{Fl}$ & suelo arcilloso \\
\hline CALABOCILLO & Sobralia sp. 1 & Quebrada & 3 & 1900 & -- & $\mathrm{Te}$ & $\mathrm{Fl}$ & tronco caído \\
\hline LAS GRUTAS & Stelis purpurea & P1 & 3 & 2860 & 19 & Еp & $\mathrm{Fl}$ & "lalush" \\
\hline PINDOE & Stelis sp. 1 & P2 & 3 & 2868 & 18 & Ep & $\mathrm{Fl}$ & "huayo" Piptadenia sp. \\
\hline PINDOE & Stelis sp. 2 & P2 & 2 & 2868 & 18 & Ep & $\mathrm{Fl}$ & "huayo" Piptadenia sp. \\
\hline PEROLITOS & Stelis sp. 3 & $\begin{array}{l}\text { Ruinas } \\
\text { Perolitos }\end{array}$ & 2 & -- & -- & Ep & $\mathrm{Fl}$ & tronco caído \\
\hline LAS GRUTAS & Trichopillia fragans & P1 & 2 & 2500 & -- & Еp & $\mathrm{Fl}$ & tronco caído \\
\hline PAN DE AZUCAR & Trichopillia sp. 1 & $\mathrm{~T} 1$ & 2 & 2164 & 14 & Еp & $\mathrm{iFl}$ & tronco caído \\
\hline CALABOCILLO & Xylobium sp. 1 & P7 & 3 & 2189 & 14 & $\mathrm{Te}$ & $\mathrm{Fl}$ & tronco caído \\
\hline
\end{tabular}

Hábito: Ep:Epifita, Te: Terrestre. Fenología: Fl: Floración, iFl: Inicio de floración, fFl: Fin de floración, Fr: Fructificación 
Frecuencia.

La frecuencia de una especie se puede definir como la proporción de unidades muestrales en que está presente en relación al número total de unidades muestrales, expresada en porcentaje. Esta definición fue aplicada a nivel de género (frecuencia del género), $\mathrm{y}$ los valores son siempre relativos y altamente dependientes de la dimensión y forma de las unidades de muestreo, se consideró realizar el análisis de parcelas independiente al de los transectos. Esta frecuencia permite representar la uniformidad de la distribución y la amplitud de nicho del género, en el espacio de referencia y la probabilidad de encontrarlo.

\section{Resultados y discusión.}

Riqueza de orquídeas

Producto de esta evaluación preliminar de orquídeas dentro de la zona comprendida por el PNC y la zona propuesta para su ampliación, se registraron un total de 88 morfoespecies. Así, durante el mes de febrero se registraron 53 orquídeas distribuidas dentro del sector de San Andrés de Cutervo, y en marzo se registraron 35 nuevas morfoespecies dentro del sector de Santo Domingo de la Capilla, siendo 16 morfoespecies de orquídeas comunes a ambos sectores.

Para una determinación apropiada de orquídeas las flores son imprescindibles, por lo que, se determinaron 56 orquídeas encontradas en estado de floración (que representan el $63.6 \%$ del total registrado) repartidas en 16 géneros, y de las cuales, 11 fueron determinadas a nivel de especie (Tabla 2). Los géneros Pleurothalis, Stelis, Maxillaria y Epidendrum presentan las mayores frecuencias, siendo los más numerosos y diversos. El restante $36.4 \%$ de orquídeas no pudieron ser determinadas por no encontrarse en estado de floración durante la época de muestreo.

Tabla 3. Frecuencia de los géneros para la evaluación por Cuadrantes.

\begin{tabular}{clccc}
\hline & Morfo & $\begin{array}{c}\text { Cuadrantes } \\
\text { donde esta } \\
\text { presente el } \\
\text { género }\end{array}$ & $\begin{array}{c}\text { Frecuencia } \\
\text { del género } \\
\text { (\%) }\end{array}$ \\
\hline 1 & Barbosella & 2 & 2 & 29 \\
2 & Epidendrum & $\mathbf{6}$ & $\mathbf{4}$ & $\mathbf{5 7}$ \\
3 & Fernandezia & 2 & 2 & 29 \\
4 & Lycaste & 1 & 1 & 14 \\
5 & Masdevallia & 1 & 1 & 14 \\
6 & Maxillaria & $\mathbf{5}$ & $\mathbf{4}$ & $\mathbf{5 7}$ \\
7 & Odontoglosum & 2 & 2 & 29 \\
8 & Otoglosum & 1 & 2 & 29 \\
9 & Pleurothalis & $\mathbf{9}$ & $\mathbf{5}$ & $\mathbf{7 1}$ \\
10 & Stelis & $\mathbf{3}$ & $\mathbf{5}$ & $\mathbf{7 1}$ \\
11 & Trichopillia & 2 & 2 & 29 \\
12 & Xylobium & 1 & 1 & 14 \\
\hline & Total & $\mathbf{3 5}$ & & \\
\hline
\end{tabular}

Tabla 4. Frecuencia de los géneros para la evaluación por transectos.

\begin{tabular}{clccc}
\hline & Género & $\begin{array}{c}\text { Morfo } \\
\text { especies }\end{array}$ & $\begin{array}{c}\text { Transectos } \\
\text { donde está } \\
\text { presente el } \\
\text { género }\end{array}$ & $\begin{array}{c}\text { Frecuencia } \\
\text { del género } \\
\text { (\%) }\end{array}$ \\
\hline 1 & Barbosella & 1 & 1 & 9 \\
2 & Elleanthus & 2 & 2 & 18 \\
3 & Epidendrum & $\mathbf{9}$ & $\mathbf{1 0}$ & $\mathbf{9 1}$ \\
4 & Fernandezia & 1 & 1 & 9 \\
5 & Lockharthia & 1 & 1 & 9 \\
6 & Lycaste & 1 & 3 & 27 \\
7 & Maxillaria & $\mathbf{7}$ & $\mathbf{8}$ & $\mathbf{7 3}$ \\
8 & Odontoglosum & 1 & 3 & 27 \\
9 & Oncidium & 1 & 1 & 9 \\
10 & Pleurothalis & $\mathbf{7}$ & $\mathbf{9}$ & $\mathbf{8 2}$ \\
11 & Stelis & $\mathbf{4}$ & $\mathbf{9}$ & $\mathbf{8 2}$ \\
12 & Trichopillia & 1 & 1 & 9 \\
\hline & Total & $\mathbf{3 6}$ & & \\
\hline
\end{tabular}

Abundancia de especies

El análisis de la densidad de especies para las orquídeas determinadas (56), muestra que el 21.4\% son raras, el $25 \%$ son ocasionales, el $42.9 \%$ son frecuentes, el 7.1\% son abundantes y el 3.6\% son muy abundantes.

El análisis de la frecuencia del género para el muestreo por cuadrados, revela frecuencias de $71 \%$ para los géneros Pleurothallis y Stelis y de 57\% para los géneros de Epidendrum y Maxillaria (Tabla 3). Para la evaluación del muestreo por transectos se integraron los datos de los transectos pareados, el género Epidendrum muestra la frecuencia más alta con 91\%, seguido por Pleurothallis y Stelis con $82 \%$ y Maxillaria con $73 \%$ (Tabla 4). Ambos tipos de muestreo determinan que los géneros Pleurothalis, Epidendrum, Maxillaria y Stelis son los más frecuentes y mejor distribuidos dentro del área de estudio (Figura 1).

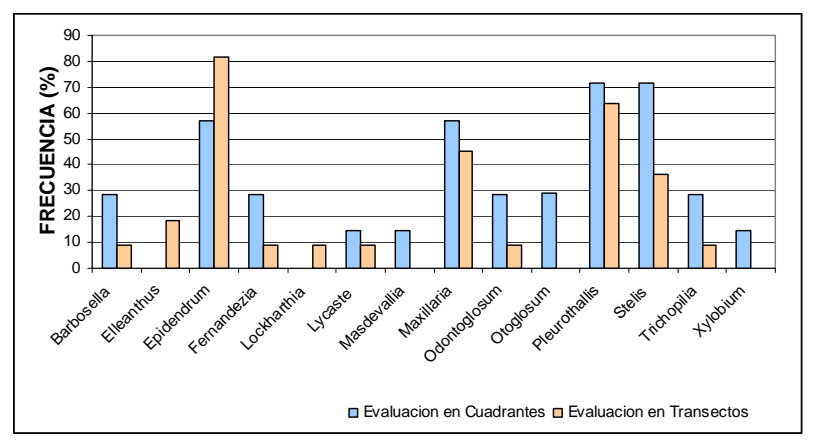

Figura 1. Frecuencias de los géneros determinados de orquídeas.

Distribución espacial de orquídeas

Los géneros Pleurothalis, Maxillaria, Epidendrum y Stelis son los más abundantes y mejor distribuidos 
en toda el área de estudio. En tanto, los géneros Barbosella, Fernandezia y Oncidium sólo se reportan para el área que comprende el PNC; Lockharthia, Lycaste, Masdevallia y Xylobium se distribuyen tan solo dentro del área de la propuesta de ampliación del PNC, y los géneros Sobralia y Elleanthus en algunos sectores de la zona de amortiguamiento.

A nivel de especie, las orquídeas muestran una distribución particular en la zona de estudio. Dentro del sector San Andrés de Cutervo Elleanthus strobillifer se distribuye de manera abundante entre el pajonal rocoso del subsector La Chira y Elleanthus sp.1 se distribuye alrededor de la Laguna la Chira.

Oncidium macranthum y Fernandezia subbiflora se distribuyen a 2 900msnm en los pocos árboles observados en una pradera de formación secundaria existente entre los subsectores de la Chira y la Pucarilla. Fernandezia ionanthera fue ubicada en el subsector Shitabamba y Trichopillia fragans en el subsector Las Grutas. Dentro del sector de Santo Domingo de la Capilla, Trichopillia sp.1 se distribuye abundantemente en el subsector Calabocillo y Odontoglosum sp.1 es abundante en los subsectores La Florida y San Pedro. En la zona de amortiguamiento, en una de las quebradas entre los subsectores de La Florida y Cedropampa, Sobralia rosea se distribuye abundantemente, encontrándose plantas de hasta $3 \mathrm{~m}$ de altura.

La morfoespecie Stelis sp.2 muestra una amplia distribución, ubicándose en 8 de los 13 subsectores evaluados, como son Shitabamba, Pindoé, Bañadera del oso, San Pedro, Calabocillo, La Florida, Pan de azúcar y Perolitos.

Orquideario.

De las 88 morfoespecies de orquídeas observadas se colectaron 84, que fueron encontradas sobre ramas, troncos o árboles muertos en las zonas de bosque destruidos debido a la tala que se da en ciertos sectores del área de estudio. Estas orquídeas no hubieran podido sobrevivir en tales condiciones de destrucción de su hábitat por lo que se estableció un orquideario dentro de las instalaciones del INRENA del distrito de San Andrés de Cutervo, de cuyo mantenimiento esta a cargo el personal de guardaparques del ANP.

Fichas Botánicas.

Para mantener un registro más detallado de las 11 especies determinadas se elaboraron sus respectivas fichas botánicas donde se detallan los datos de colección y la descripción botánica de las plantas. El modelo de ficha empleado es la Ficha Botánica del Herbario MOL, modificado por los autores para este estudio (Figura 2).

\section{Conclusiones y recomendaciones.}

Se determinaron un total de 88 morfoespecies de orquídeas presentes en el área del Parque Nacional Cutervo y la propuesta para su ampliación. La riqueza observada fue parcial, por lo que es necesario establecer unidades de muestreo más representativas de los tipos de bosques y mejor distribuidas en la zona. También, se debe tomar en consideración contar con un equipo básico (estereoscopio y cámara fotográfica) y bibliografía especializada para la identificación de las orquídeas antes de ser preservadas, para lograr un inventario más representativo.

De acuerdo a la metodología planteada, los géneros Pleurothalis, Epidendrum, Maxillaria y Stelis son los más frecuentes.

El orquideario establecido puede considerarse como una herramienta para el manejo ex situ de orquídeas, que permita generar información necesaria para su aclimatización, cultivo y reproducción.

Se recomienda realizar estudios más específicos que consideren los variados nichos ecológicos presentes en el área, para una estimación más precisa de la riqueza de especies de orquídeas.

Debido al riesgo de destrucción de los bosques de neblina por factores antropogénicos como la ampliación de la frontera agrícola y tala de

$\quad$ FICHA BOTÁNICA No. 10
Datos de colección
Fecha de colección: $13103 / 04$
Departamento.: Cajamarca
Provincia: Cutervo
Sector: Santo Domingo de la Capilla
Subsector: Pán de azúcar
Colector (es): María Sánchez, Agustín Vásquez
Nombre científico: Trichopillia sp.
Código: MASp 68
Altitud: 2164 m.s.n.m.
Hábitat: Bosque Húmedo
Hábito: Epífita baja
Fenologia: Floración
Abundancia: Escasa
Caracteristicas botánicas
Epífita. Planta hasta $18 \mathrm{~cm}$. de alto.
Rizoma corto de seudobulbos apretados.
Seudobulbos unifoliados, oblongos, semiaplanados, de 4.8 x $1.8-2.5 \mathrm{~cm} .$, protegidos
en su base por un par de brácteas escariosas acuminadas.
Hojas elíptico lanceoladas acuminadas, conduplicadas en su base, de 8-15 x 2.7-4
cm.
Inflorescencia basal axilar, un pedúnculo recto de $10-18 \mathrm{~cm}$. de alto sale de una
bráctea basal con $2-4$ flores.
Flor de pétalos y sépalos blancos.

Figura 2. Ficha Botánica del Herbario MOL modificada por los autores. 
subsistencia, urge un estudio mas intenso de colección e identificación de la diversidad real existente, ya que estos bosques son considerados muy ricos en diversidad de orquídeas.

\section{Agradecimientos.}

Se agradece a la Ing. Agr. Jessica Alarcón Ramírez, responsable de la administración del Parque Nacional Cutervo, y su personal de guardaparques conformado por Bach. Hernán Chávez, Tco. Víctor León, Tco. Agustín Vásquez y Tco. José Zaldívar, por su apoyo en la ejecución del presente estudio. Así mismo, al Blgo. Marco León, especialista del INIA San Martín, por su apoyo en la identificación de orquídeas; y al Bach. Luis Alza León, por su orientación en el análisis de los datos.

\section{Literatura citada}

Bennett Jr. D. \& Christenson E. 1993. Icones Orchidacearum Peruvianum. Plates 1-200.

Bennett Jr. D. \& Christenson E. 1995. Icones Orchidacearum Peruvianum. Plates 201-400.

Cavero M., Collantes B. \& Patroni C. 1991. Orquídeas del Perú. Centro de Datos para la Conservación del Perú.

Collantes B. 1998. Orquídeas del Perú en peligro. Rumbos.III 13:6.

Collantes-Salazar J. 2003. Estudio de distribución e identificación de palmeras blancas y helechos gigantes del Parque Nacional Cutervo y propuesta de ampliación, Cajamarca-Perú. INRENA, Proyectos STEM.

Dodson C.H. \& Bennett D. 1989. Icones Plantarum Tropicarum: Orchids of Peru. Serie II - Fascículo I y II.

Dodson C.H., Chase M.W., Luer C.A. 1987. Orchids of Ecuador. The Genera Missouri Botanical Garden, St Lois. Vol 1. IUCN/SSC Orchid Specialist Group Ed. Scribd.

Graf E. \& Sayagués Laso L. 2000. Muestreo de la vegetación. Unidad de sistemas ambientales, Facultad de Agronomía, Universidad de la Republica - Montevideo, Uruguay.

López- Miranda A. 1971. Nota Botánica sobre el Parque nacional de Cutervo. Boletín de la Sociedad Botánica de La Libertad, Trujillo- Perú. Bol. Soc. Bot. La Libertad 3(1): 71-74.

Mendoza Ocampo A. 1996. ABISEO - Patrimonio Mundial en Emergencia. Moyabamba, Perú. Segunda Edición.

Schweinfurth Ch. 1959. Orchids of Peru. Fieldiana Botany. $30(2)$.

Schweinfurth Ch. 1960. Orchids of Peru. Fieldiana Botany. 30(3).

Schweinfurth Ch. 1961. Orchids of Peru. Fieldiana Botany. 30(4).

Schweinfurth Ch. 1970. First supplement to the Orchids of Peru. Chicago, Field Museum of Natural History. Vol 33. December 31: 35-40.

\footnotetext{
${ }^{1}$ María Sánchez Recuay. Bachiller en Biología. UNALM. maesanchezr@gmail.com

${ }^{2}$ Abelardo Calderón Rodríguez. Prof. Principal de Biología. UNALM. acalderon@lamolina.edu.pe
} 